Agric. Biol. Chem., 41 (4), 631 634, 1977

\title{
Studies on Action Pattern of Amylase-catalized Hydrolysis of Amylose Using TNS Fluorescence as a Probe
}

\author{
Hitoshi Kondo, Hiroshi NaKatani and Keitaro Hiromi \\ Laboratory of Enzyme Chemistry, Department of Food Science and Technology, \\ Faculty of Agriculture, Kyoto University, Sakyo-ku, Kyoto, Kyoto 606 \\ Received October 6, 1976
}

\begin{abstract}
A large fluorescence enhancement of 2-p-toluidinylnaphthalene-6-sulfonate (TNS) caused by amylose decreases as the substrate is degraded by amylases. This property was used to follow the enzymatic hydrolysis of amylose and analyze the action pattern of six kinds of amylases. This method can substitute the conventional blue value method, and is more sensitive for short chain amylodextrins. The advantage of the new method over the blue value method is that it is useful to monitor the hydrolysis of maltooligosaccharides to which the blue value method cannot be applicable, and that it enables continuous monitoring of the enzyme reactions.
\end{abstract}

So far five methods have been mainly employed for following hydrolytic reaction of amylose; namely, 1) reducing power, 2) blue value of iodine-iodide complex, 3) polarimetric, 4) viscometric, and 5) blue-starch methods. In the course of the study on interaction between 2-p-toluidinylnaphtalene6 -sulfonate (TNS) and cyclodextrins, we found that linear polysaccharides such as amylose also enhance TNS fluorescence. ${ }^{1 !}$ Since small oligosaccharides show only limited fluorescence enhancement, ${ }^{1)}$ this property may possibly be used to follow the hydrolysis of amylose. In this paper, we aimed to examine this possibility and to establish a novel fluorescence method for the study on action pattern of amylases, in place of the conventional blue value method.

\section{MATERIALS AND METHODS}

Materials. TNS and crystalline Taka-amylase A (TAA) (EC 3.2.1.1) are the same as those described in the previous paper. ${ }^{1)}$ Glucoamylase (EC 3.2.1.3) from Rhizopus niveus (Lot No. 4S17, pure grade), bacterial liquefying $\alpha$-amylase (BLA) (EC 3.2.1.1, Lot

Abbreviations used: TNS, 2-p-toluidinylnaphthalene-6-sulfonate; TAA, Taka-amylase A; BLA, bacterial liquefying $\alpha$-amylase; BSAm, bacterial saccharifying $\alpha$-amylase; PPA, porcine pancreatic $\alpha$ amylase; $\overline{\mathrm{DP}_{\mathrm{n}}}$, number-average degree of polymerization.
No. 3T15, $3 \times$ crystallized), and bacterial saccharifying $\alpha$-amylase (BSAm) (EC 3.2.1.1, Lot No. 1X26, $2 \times$ crystallized) were purchased from Seikagaku Kogyo Co., Ltd., and used without further purification. The enzyme concentrations were determined spectrophotometrically at $280 \mathrm{~nm}$, assuming $E_{1 \%}^{1 \mathrm{~cm}}=14.2$ and a molecular weight of $\left.58,000^{2}\right)$ for glucoamylase, $E_{1 \%}^{1 \mathrm{~cm}}=$ 25.6 and a molecular weight of $47,500^{3}$ for BLA, and $E_{1 \%}^{1 \mathrm{~cm}}=19.8^{4)}$ and a molecular weight of $41,900^{5)}$ for BSAm. Porcine pancreatic $\alpha$-amylase (PPA) (EC 3.2.1.1, Lot No. 33C8240, $2 \times$ crystallized) was purchased from Sigma Chemical Co., and used without further purification. The enzyme concentration was determined spectrophotometrically at $280 \mathrm{~nm}$, assuming $E_{1 \%}^{1 \mathrm{~cm}}=24.0^{8)}$ and a molecular weight of $52,000 .^{7)}$ Crystalline $\beta$-amylase (EC 3.2.1.2) from soy bean was a generous gift from Mr. T. Suganuma and its concentration was determined spectrophotometrically at $280 \mathrm{~nm}$, assuming $E_{1 \%}^{1 \mathrm{~cm}}=17.3$ and a molecular weight of $\left.65,000 .{ }^{8}\right)$

Potato amylose (the number-average degree of polymerization $(\overline{\mathrm{DP}})=600$, Lot No. MOR4819) was purchased from Sigma Chemical $\mathrm{Co}$. and used without further purification.

Other chemicals were of reagent grade and used without further purification.

Methods. Fluorescence measurement was made at $25^{\circ} \mathrm{C}$ with a Hitachi MPF-2A Spectrofluorometer. The excitation wavelength of TNS-amylose system was $366 \mathrm{~nm}$ and the emission was observed at $460 \mathrm{~nm}$.

The enzyme reactions were all carried out at $25^{\circ} \mathrm{C}$. Following buffer systems were used for each amylase reaction: TAA, $0.08 \mathrm{~m}$ sodium acetate buffer ( $\mathrm{pH} 5.3$ ); BLA, $0.08 \mathrm{M}$ sodium acetate buffer ( $\mathrm{pH} \mathrm{5.5);} \mathrm{BSAm}$

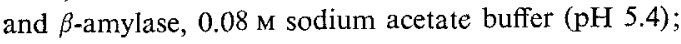


PPA, $0.02 \mathrm{M}$ sodium glycerophosphate buffer containing $0.01 \mathrm{M}$ calcium chloride ( $\mathrm{pH}$ 6.9); glucoamylase, $0.05 \mathrm{M}$ acetate buffer ( $\mathrm{pH} 4.5$ ). At appropriate intervals, $4 \mathrm{ml}$ of reaction mixture was withdrawn and put into $4 \mathrm{ml}$ of $0.08 \mathrm{~N} \mathrm{NaOH}$ solution to stop the reaction. This solution (solution A) was divided into three parts for measurements of the fluorescence, the blue value, and the reducing value. For the fluorescence measurement, $4 \mathrm{ml}$ of solution A was mixed with $4 \mathrm{ml}$ of TNS stock solution (final concentration $(4.10 \sim 6.06) \times$ $\left.10^{-4} \mathrm{M}\right)$. For the blue value measurement, $1 \mathrm{ml}$ of solution A was added to $6 \mathrm{ml}$ of $\mathrm{I}_{2}-\mathrm{KI}$ solution $(5 \times$ $10^{-4} \mathrm{M} \mathrm{I}_{2}$ and $5 \times 10^{-3} \mathrm{M} \mathrm{KI}$ ) and after filling up to $20 \mathrm{ml}$ the absorbance at $550 \mathrm{~nm}$ was measured. The reducing value was determined by the modified Somogyi-Nelson method. ${ }^{9}$ The total concentration of amylose was determined by phenol-sulfuric acid method. ${ }^{10)}$ From these values the percentage of hydrolysis was obtained.

\section{RESULTS AND DISCUSSION}

A large fluorescence enhancement was observed when TNS was added to the solution of amylose, whose spectra are shown in Fig. 1. However, the nature of the interaction between amylose and TNS may not be readily inferred as in the case of cyclodextrin-TNS

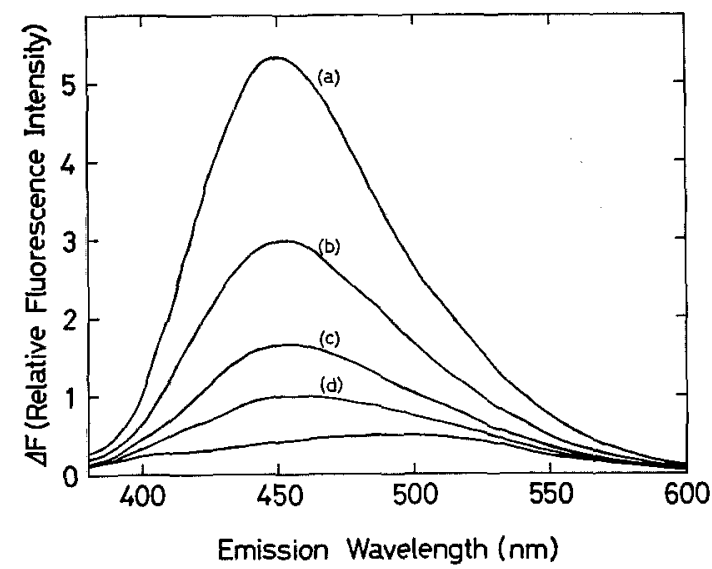

FIG. 1. Fluorescence Spectra of TNS in the Presence of Amylose $(\overline{\mathrm{DP}} \overline{\mathrm{n}}=600)$.

$[\mathrm{TNS}]=2.18 \times 10^{-4} \mathrm{M}$. Excitation wavelength $=366$ nm. Amylose concentrations: (a) 0.075 , (b) 0.038 , (c) 0.019 , and (d) $0.009 \%$. The lowest curve shows the fluorescence spectrum of free TNS in the absence of amylose. The buffer was $0.08 \mathrm{M}$ sodium acetate buffer ( $\mathrm{pH} \mathrm{5.3)} \mathrm{and} \mathrm{the} \mathrm{fluorescence} \mathrm{measurement} \mathrm{was}$ performed at $25^{\circ} \mathrm{C}$ with a Hitachi MPF-2A Spectrofluorometer. complexes. ${ }^{13}$ Since it is accepted that amylose can form helix in solution, ${ }^{11 \sim 15}$; TNS may well be included in the helical structure of amylose. However, the possibility that TNS interacts with non-helical part of amylose may not be excluded.

The results of the application of the fluorescence method for monitoring the amylasecatalyzed hydrolysis of amylose are shown in Fig. 2. In this figure action patterns of six kinds of amylases are illustrated in fluorescence intensity and blue value versus reducing value (percentage of bond split) plots. The fluorescence intensity of TNS in the reaction mixture monotonously decreases as the hydrolysis proceeds, in a similar manner to the blue value. When compared at a given percentage of hydrolysis, the fractional fluorescence intensity $(\%)$ is larger than the blue value $(\%)$. This tendency is more remarkable with endoamylases (TAA, BLA, BSAm, and PPA) than with exo-amylases (glucoamylase and $\beta$-amylase). This discrepancy between fluorescence and iodine-iodide blue color may be due to the different dependence of the two properties upon the chain length of amylose fragments. Qualitatively speaking, the minimum necessary chain length must be larger for the development of blue value than the enhancement of TNS fluorescence. First important difference between the blue value and fluorescence method is that latter may be applicable to monitor the hydrolysis of maltodextrins such as maltoheptaose. In the previous paper, ${ }^{1 /}$ we found that the magnitudes of fluorescence enhancement per glucose residue of maltoheptaose, maltotriose, maltose, and glucose were about $1100,26,12$, and 1 , respectively. On the other hand, with maltodextrins up to maltooctaose, no appreciable iodine-iodide staining were detected visually. ${ }^{16)}$

Next we tried to make a rough simulation of the course of $\alpha$-amylase-catalyzed hydrolysis observed by present fluorescence method and by the blue value method, based on the following assumptions: 1) The amylase degrades amylose in completely random fashion. 2) Oligosaccharides produced during the reaction 

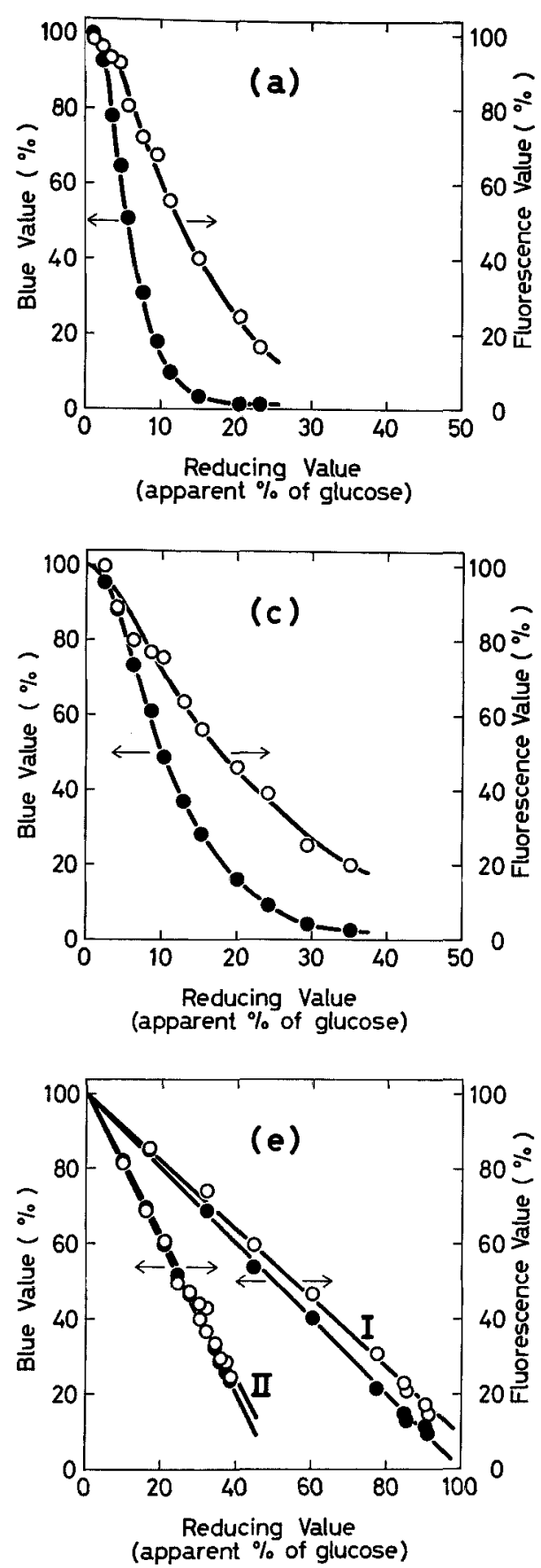

whose $\overline{\mathrm{DP}_{\mathrm{n}}}$ is larger than a critical value $(\mathrm{m})$ contribute, to the same extent (irrespective to the $\left.\overline{\mathrm{DP}_{\mathrm{n}}}\right)$, to the fluorescence enhancement or the blue value. 3) Oligosaccharides whose $\overline{\mathrm{DP}_{\mathrm{n}}}$ 's do not exceed the critical value $(m)$ do not contribute to either at all. The computer
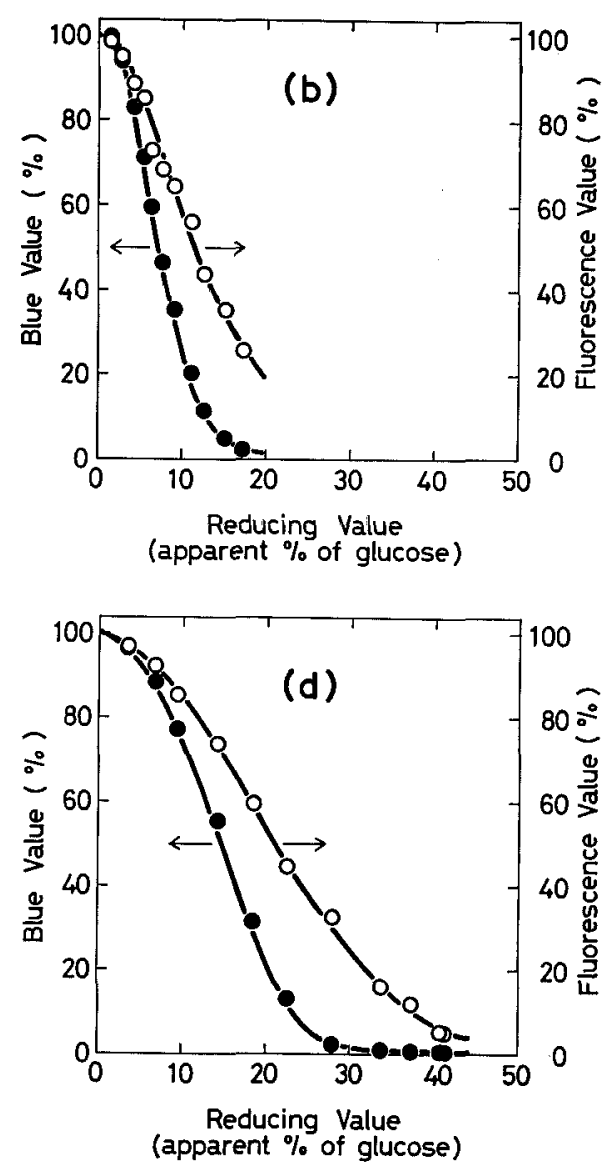

FIG. 2. Action Patterns of Amylase-catalyzed Hydrolysis of Amylose $\left(\overline{\mathrm{DP}_{\mathrm{n}}}=600\right)$.

All enzyme reactions were carried out at $25^{\circ} \mathrm{C}$ and amylose concentration was $0.15 \%$ Enzyme concentrations and buffer systems were as follows: (a) [TAA] $=3.40 \times 10^{-9} \mathrm{M}, 0.08 \mathrm{M}$ sodium acetate buffer (pH 5.3); (b) [BLA] $=2.43 \times 10^{-2} \mathrm{M}, 0.08 \mathrm{M}$ sodium acetate buffer (pH 5.5); (c) [BSAm] $=8.56 \times 10^{-9} \mathrm{M}$, $0.08 \mathrm{M}$ sodium acetate buffer (pH 5.4$)$; (d) [PPA] = $6.40 \times 10^{-8} \mathrm{M}, 0.02 \mathrm{M}$ sodium glycerophosphate buffer containing $0.01 \mathrm{M}$ calcium chloride ( $\mathrm{pH} 6.9$ ); (e)-I [glucoamylase] $=3.55 \times 10^{-7} \mathrm{M}, 0.05 \mathrm{M}$ acetate buffer $(\mathrm{pH} 4.5) ;(\mathrm{e})$-II $[\beta$-amylase $]=2.30 \times 10^{-8} \mathrm{M}, 0.08 \mathrm{M}$ sodium acetate buffer $(\mathrm{pH}$ 5.4). For fluorescence measurements, see METHODS. TNS concentrations were as follows: (a), $4.66 \times 10^{-4} \mathrm{M}$; (b) and (c), $4.77 \mathrm{X}$ $10^{-4} \mathrm{M}$; (d), $4.10 \times 10^{-4} \mathrm{M}$; (e)-I, $6.06 \times 10^{-4} \mathrm{M}$; (e)-II, $4.66 \times 10^{-4} \mathrm{M}$. For blue value measurement, see METHODs. $\mathrm{O}-\mathrm{O}$, fluorescence value versus reducing value; - blue value versus reducing value.

programme was devised by Dr. R. Matsuno et al. ${ }^{17)}$ Reasonable agreement with the experimental results was obtained for TAA and BLA with $m=12 \sim 15$ in the fluorescence value-reducing value profile, and $m=25 \sim 30$ in the blue value-reducing value profile (Fig. 3). 


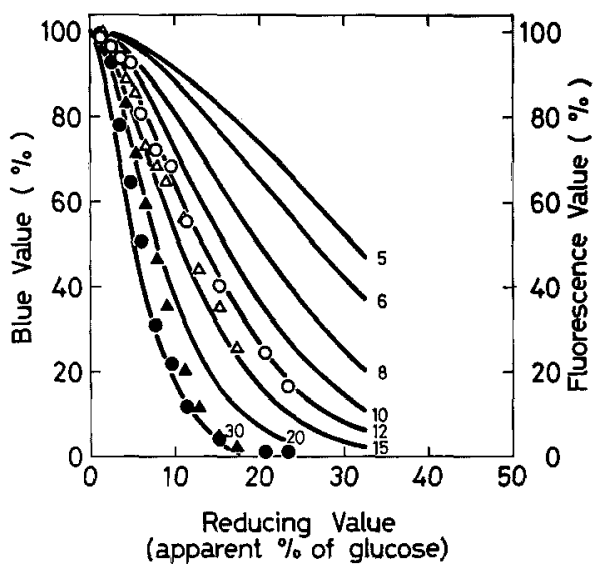

FIG. 3. Theoretical Action Pattern of Hypothetical Amylase-catalyzed Hydrolysis of Amylose ( $\overline{\mathrm{DP}_{\mathrm{n}}}=$ 600).

In the theoretical curves (solid lines), it is assumed that oligosaccharides whose $\overline{\mathrm{DP}_{n}}$ 's do not exceed the critical value $(m)$ (shown in the figure) contribute neither to the fluorescence enhancement nor the blue color development. Experimental values in the case of TAA and BLA in Fig. 2 are also shown in this figure. For TAA, $O$; fluorescence value versus reducing value, ; blue value versus reducing value. For BLA, $\triangle$; fluorescence value versus reducing value, $\Delta$; blue value versus reducing value. The other conditions were the same as in Fig. 2.

Considering the oversimplified nature of the above assumptions, the agreement may be satisfactory.

Another important difference between the blue value and the fluorescence method is that the fluorescence method may be used for continuous monitoring of amylase-catalyzed hydrolysis of amylose, ${ }^{18,19)}$ whereas the blue value method is restricted only to a discontinuous one because of strong inhibition of amylase activity by iodine. ${ }^{20 \text {, }}$

Acknowledgment. We would like to express our gratitude to Associate Professor A. Maeda of the Department of Chemistry, Faculty of Science, Kyoto University for allowing us to use the spectrofluorometer. We also wishes to express our thanks to Sankyo Co., Ltd. for their kind supply of "Takadiastase Sankyo."

\section{REFERENCES}

1) H. Kondo, H. Nakatani and K. Hiromi, J. Biochem., 79, 393 (1976).

2) Y. Tsujisaka, personal communication.

3) T. Isemura and K. Kakiuchi, J. Biochem., 51, 385 (1962).

4) A. Nishida, J. Fukumoto and T. Yamamoto, Agric. Biol. Chem., 31, 682 (1967).

5) H. Toda and K. Narita, J. Biochem., 63, 302 (1967).

6) J. Hsiu, E. Fischer and E. Stein, Biochemistry, 3, 61 (1964).

7) P. Zavodszky and P. Elödi, Acta Biochem. Biophys. Acad. Sci. Hung., 5, 225 (1970).

8) A. Gertler and Y. Birk, Biochem. J., 95, 621 (1965).

9) K. Hiromi, Y. Takasaki and S. Ono, Bull. Chem. Soc. Jpn., 36, 563 (1963).

10) M. Dubois, K. A. Gilles, J. K. Hamilton, P. A. Rebers and F. Smith, Anal. Chem., 28, 350 (1956).

11) T. Kuge and S. Ono, Bull. Chem. Soc. Jpn., 34, 1264 (1961).

12) W. Banks, C. T. Greenwood and K. M. Khan, Carbohyd. Res., 17, 25 (1971).

13) K. Takeo and T. Kuge, Agric. Biol. Chem., 33, 1174 (1969).

14) K. Takeo and T. Kuge, ibid., 34, 568 (1970).

15) K. Takeo and T. Kuge, ibid., 34, 1787 (1970).

16) I. A. Thoma and D. French, J. Am. Chem. Soc., 82, 4144 (1960).

17) R. Matsuno, T. Suganuma, H. Fujimori, K. Nakanishi, K. Hiromi and T. Kamikubo, submitted to J. Theor. Biol.

18) H. Kondo, Master's Thesis, Kyoto University (1975).

19) K. Kurahashi and A. Ishihara, Seikagaku, 48, 552 (1976).

20) T. Yamamoto, Bull. Agric. Chem. Soc., Jpn., 19, 121 (1955). 